\title{
Evaluación y estudio comparativo mediante modelos sistémicos de la implantación del sistema APPCC aplicado al sector agroalimentario.
}

\author{
Hernandis-De Haro, Cristina ${ }^{a}$ \& Esnal-Angulo, Iñaki ${ }^{b}$ \\ ${ }^{a} \mathrm{PhD}$ candidate. Universitat Politècnica de València, Spain. crissthernha@gmail.com. \\ ${ }^{\mathrm{b}} \mathrm{PhD}$ candidate. Universitat Politècnica de València, Spain. iaesan@doctor.upv.es
}

\section{Resumen}

El Reglamento comunitario (CE) $n^{\circ} 852 / 2004$, relativo a la higiene de los productos alimenticios, establece que las empresas alimentarias deben cumplir con los requisitos obligatorios de desarrollar, aplicar y mantener un procedimiento permanente basado en los principios del sistema APPCC (Analisis de los Puntos Críticos de Control).

Actualmente, se encuentran implantados los sistemas basados en APPCC en la mayoría de empresas del sector agroalimentario. No obstante, en muchos casos la implantación del sistema no se realiza correctamente, siendo inefectiva y no garantizando la seguridad alimentaria. El objetivo de este trabajo se centra en demostrar que la introducción de los modelos sistémicos aplicados en la optimización de sistemas jerarquizados, puede mejorar la implantación y efectividad de los sistemas APPCC en el sector agroalimentario, asi como detectar los posibles fallos en dicho sistema, y por tanto, garantizar adecuadamente la producción de alimentos seguros. Para ello, se ha llevado a cabo un estudio retrospectivo de índole cualitativo, en cuatro industrias pertenecientes al sector agroalimentario, obteniendo datos relativos a documentos y registros generados por el propio sistema de autocontrol, actas de inspección derivadas del control oficial, analíticas microbiológicas y verificaciones internas. Se ha determinado, en los casos estudiados, que se producen fallos en cuanto aplicación de procedimientos, toma de registros, interpretación de medidas correctoras a llevar a cabo y falta de vigilancia de PCC identificados, en cuyo caso perjudica los intereses productivos de la empresa alimentaria y no garantiza una correcta protección de la salud de los consumidores. Finalmente, la evaluación mediante modelos sistémicos del APPCC permite detectar los fallos de implantación en las industrias implicadas y ofrecer una herramienta, tanto para los operadores de empresas alimentarias, como para los servicios de control oficial, que permite garantizar una correcta aplicación del sistema de autocontrol.

Palabras clave: $A P P C C$, modelos sistémicos, seguridad alimentaria, sector agroalimentario. 


\begin{abstract}
Community Regulation (CE) No 852/2004 on the hygiene of foodstuffs provides that food businesses must comply with the mandatory requirements to develop, implement and maintain a permanent procedure based on the principles of HACCP (Hazrd Analysis Critical Control Point) system points.

Currently, are implemented HACCP-based systems in most food businesses. However, in many cases the implementation of the system is not successful, being ineffective and not ensuring food security.

The aim of this work is focused on demonstrating that the introduction of systemic models applied in optimizing hierarchical systems can improve the implementation and effectiveness of HACCP systems in the food sector and detect possible failures in the system, and therefore adequately ensure safe food production.

To this end, has conducted a retrospective study of qualitative nature, in four industries belonging to the food sector, obtaining data on documents and records generated by the self-monitoring system itself, inspection reports derived from official control, microbiological analysis and internal verification.

It has been determined in the case studied, which faults occur as application procedures, making records, interpretation of correctives to carry out measures and lack of monitoring of HACCPs identified, in which case it damages the productive interests of the food business and does not guarantee proper protection of the health of consumers.

Finally, the evaluation of HACCP by systemic models detects failures of implementation in the industries concerned and provide a tool for both food business operators and for services official control, which helps to ensure a successful implementation of self-monitoring system .
\end{abstract}

Keywords: $H A C C P$, systemic models, food security, food sector.

\title{
1. Introducción
}

En primer lugar analizaremos aquellas cuestiones relativas a los APPCC (Análisis de los Puntos Críticos), cuyo conocimiento será primordial para el desarrollo de esta investigación en el Sector Alimentario en la Comunidad Valenciana.

\subsection{Situación actual de la seguridad alimentaria o marco de referencia}

En marzo de 1996 surge una nueva variante de la enfermedad de Creutzfeldt-Jacob asociada a la Encefalopatía Espongiforme Bovina, lo que provoca una de las mayores crisis alimentarias de los últimos tiempos. Éste fue uno de los factores que obligó a plantearse a la Unión Europea y a sus Estados Miembros la política a seguir con respecto a la seguridad alimentaria.

La Comisión Europea adoptó, en enero de 2002, el Libro Blanco sobre la seguridad alimentaria, con la finalidad de desarrollar un marco legislativo garante de un alto nivel de seguridad alimentaria y una elevada protección de la salud de los consumidores. En enero de 2002 se publica una importante normativa, de directa aplicación, denominado: Reglamento 178/2002, del Parlamento y del Consejo, de 28 de enero, por el que se establecen principios y requisitos generales de la legislación alimentaria, 
también se crea la Autoridad Alimentaria Europea y se fijan los procedimientos relativos a la seguridad alimentaria.

El Consejo y el Parlamento europeo adoptaron un conjunto de Reglamentos y Directivas que reestructuraron y actualizaron las normas de higiene de todos los productos alimenticios, incluidos los productos de origen animal. Este conjunto normativo es conocido por el "paquete de higiene" y es aplicable desde el 1 de enero de 2006.

En dichos reglamentos se obliga a la industria alimentaria a implantar un Sistema de Autocontrol (Reglamento (CE) no 852/2004).

\subsection{Sistemas de Autocontrol}

Los sistemas de autocontrol son herramientas imprescindibles que deben aplicar los operadores alimentarios para asegurar la inocuidad de sus productos alimenticios (Suárez Iglesias, J.L. y col., 2012). Están constituidos por los requisitos previos de higiene y trazabilidad (RPHT) y el plan de análisis de peligros y puntos de control crítico (APPCC).

Consisten en "un enfoque sistemático para identificar peligros que pueden afectar la inocuidad de un alimento, a fin de establecer las medidas para controlarlos" (Martí, L.E. y col. 2012). Pueden llegar a ser más o menos complejos, desde las Guías de las Correctas Prácticas de Higiene (GCPH) hasta los manuales de APPCC.

Estos sistemas de autocontrol han de ser coherentes y adaptarse a las características particulares de cada establecimiento (Mejías Carpena, C. y col., 2009).

Evaluación del nivel de implantación de los sistemas de autocontrol

La evaluación de los sistemas HACCP son un elemento clave para asegurar la gestión eficaz de la inocuidad de los alimentos, sin embargo, según Wallace, Powell y Holyoak (2005), no existe ningún enfoque aceptado o metodología común disponible para ser utilizados por industriales, auditores u organismos reguladores (Gutiérrez, N. y col., 2011). En investigaciones realizadas por SUÁREZ IGLESIAS, J.L. y col. 2012, Suasnavas, N. y col. (2007) usaron listas de verificación/ revisión para evaluar el cumplimiento de los prerrequisitos relacionados con higiene, inocuidad y, en general, gestión de la calidad en la industria alimentaria

La legislación comunitaria en materia de higiene de los alimentos establece que la autoridad competente efectuará controles oficiales para comprobar que se cumplen los requisitos especificados, que estos controles deberán realizarse de una forma eficaz, y que uno de los métodos o técnicas a utilizar es la auditoría, concretamente "Auditoría de sistemas de autocontrol basados en los principios del APPCC" (Reglamento 882/2004 y 854/2004) (Carravilla, S. y col., 2009). Por este motivo muchos estudios para la evaluación de los sistemas de autocontrol se basan en los resultados reflejados en los formularios de inspección que utilizan los agentes de control oficial.

Toda esta legislación marca las directrices a considerar desde una nueva estrategia a considerar en la seguridad alimentaria. 
Evaluación y estudio comparativo mediante modelos sistémicos de la implantación del APPCC aplicado al sector agroalimentario.

\section{Objetivos}

En este estudio de carácter exploratorio se pretende:

- Conocer el grado de implantación de los sistemas de autocontrol de obligada exigencia dentro de las empresas alimentarias industriales

- Detectar los principales fallos en la aplicación de los sistemas de autocontrol

- Introducir los modelos sistémicos y evaluar su aplicación en la optimización de sistemas jerarquizados, para mejorar la implantación y efectividad de los sistemas APPCC.

\section{Material y Método}

Se han revisado diversos estudios, que muestran los resultados de la implantación del sistema APPCC, siendo su interés primordial detectar las variables más significativas para realizar una correcta evaluación del sistema de autocontrol, así como para la representación del modelo sistémico.

Se analiza en un primer estudio, la valoración de la implantación de los requisitos previos de higiene y trazabilidad (RPHT) en 179 comercios minoristas (Arjona, C. y col., 2009).

Se analiza en un segundo estudio la evaluación del grado de implantación de los sistemas de autocontrol en 91 industrias de harinas y derivados (Baro Duarte, J. y col., 2009)

Un tercer estudio aporta la valoración de la implantación del APPCC en diferentes sectores alimentarios de Murcia, (González Fernández, M. y col., 2007).

En otro de los estudios se analiza la evolución de la implementación de los sistemas de autocontrol en 36 comedores colectivos, (Serrano Galán, E., 2009). Un estudio sobre 15 auditorías en actividades de restauración sin registro sanitario de alimentos en un distrito de Madrid, muestran el diferente nivel de implantación de un sistema completo de APPCC (Figuras 1 y 2)

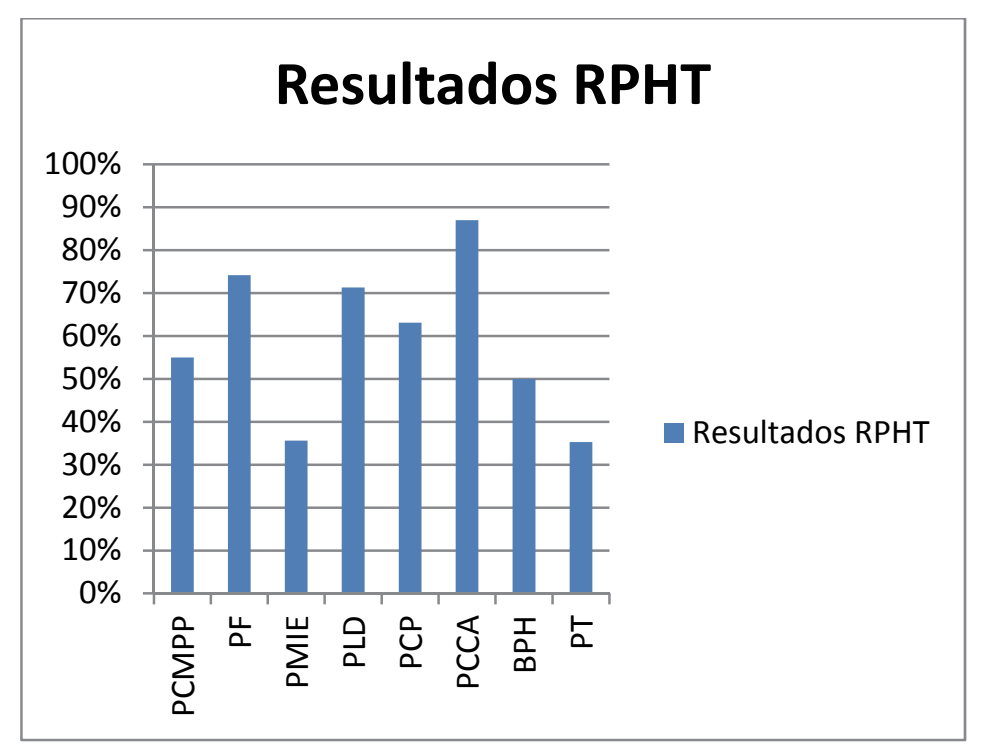

PCMPP: Plan de Control Materias Primas y Proveedores

PF: Plan de Formación

PMIE: Plan de Mantenimiento de

Instalaciones y Equipos

PLD: Plan de Limpieza y Desinfección

PCP: Plan de Control de Plagas

PCCA: Plan de Control de la Calidad del Agua

BPH: Buenas prácticas de higiene

PT: Plan de Trazabilidad

Figura 1. Resultados RPHT estudio Villar Gaspar M.R. (2009). 


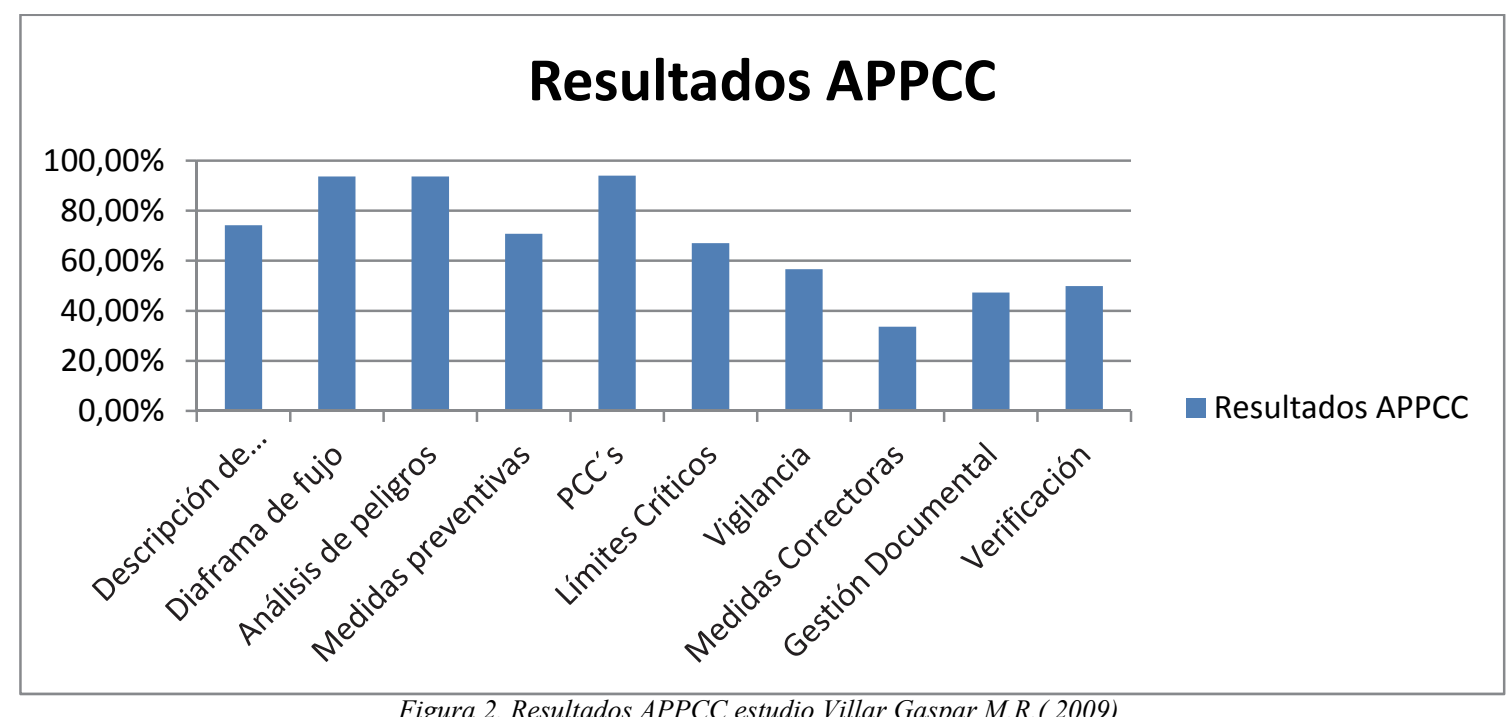

Utilizaremos los modelos sistémicos (Hernandis, B., 2003) en particular el Modelo de Formulación por Objetivos para analizar el cumplimiento de los planteamientos estratégicos, tácticos y operativos para la implantación de un sistema APPCC con objeto de analizar las posibles mejoras para el seguimiento y control en los planes de inspección alimentaria.

Procederemos a la revisión de los modelos organizacionales, describiendo sus características y elementos a determinar. Para ello se utilizará el siguiente gráfico desarrollado en la Escuela de Investigación Operativa y Sistemas de la Universidad de Valencia (2003)

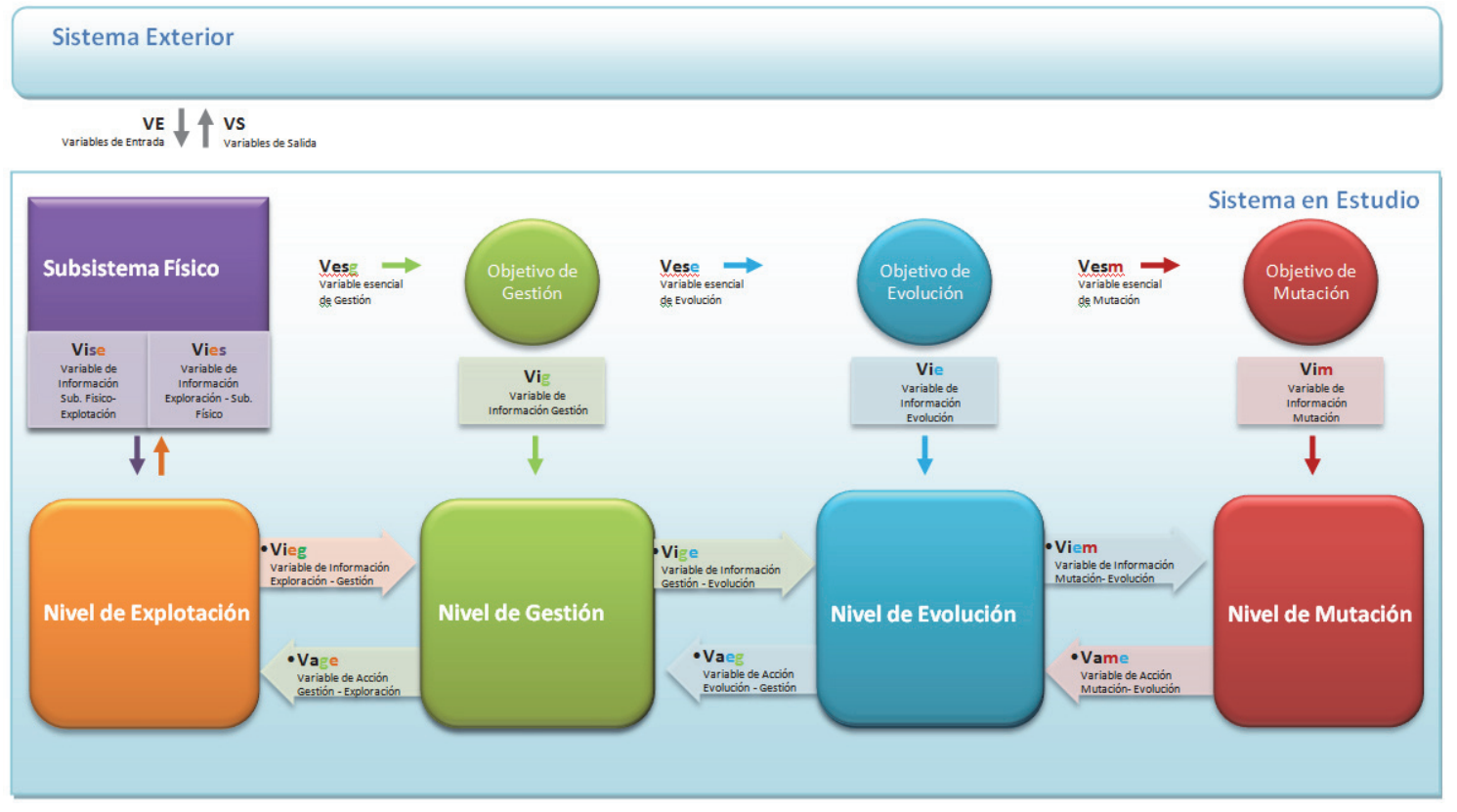

Figura 36. Modelo de Formulación por objetivos, Hernandis B. (2003) 
Para la aplicación de estos modelos el primer paso consiste en definir los límites del sistema y analizar tanto los elementos integrados en éste, como los elementos que influyen sobre el sistema en estudio y que no pueden ser controlados por éste.

El sistema en estudio se subdivide para su análisis en varios sistemas o niveles, los cuales serán a su vez subsistemas del anterior. La aplicación de los modelos sistémicos, permite un seguimiento y control de los sistemas en general independientemente de la disciplina objeto de estudio. Esto es lo que nos ha inducido a pensar en ellos, como es un modelo adecuado que permita la implementación del APPCC y su seguimiento y control posterior. El uso de estos modelos ha permitido en el campo del diseño la implementación y modelado en múltiples casos (citar algunos modelos) comprobando por tanto que desde la investigación cualitativa esta nos permite por analogía evidenciar su utilidad en esta investigación.

\section{Resultados y discusión}

En el primer estudio sobre la valoración de la implantación de los requisitos previos de higiene y trazabilidad (RPHT) en 179 comercios minoristas, se observa:

Que el 46\% de los establecimientos estudiados no poseen documentación RPHT, un 39\% poseen documentación pero esta no se encuentra implantada, y tan solo un $10 \%$ tiene documentación desarrollada e implantada. De ello cabe deducir que con el fin de adaptarse a estos pequeños comercios el sistema de autocontrol enfocado y adaptado a ellos ha de ser simplificado para su correcta implementación (Arjona, C. y col., 2009).

Del estudio realizado sobre la evaluación del grado de implantación de los sistemas de autocontrol en 91 industrias de harinas y derivados (Baro Duarte, J. y col., 2009), se obtienen los resultados representados en la tabla 1:

Tabla 1. Resultados estudio Baro Duarte, J. y col. 2009.

\begin{tabular}{|l|l|l|}
\hline Plan de Control de la Calidad del Agua & Implantados & No implantados \\
\hline Plan de Limpieza y Desinfección & 71 & 20 \\
\hline Plan de Control de Plagas & 67 & 24 \\
\hline Plan de Mantenimiento de Instalaciones y Equipos & 58 & 33 \\
\hline Plan de Trazabilidad & 68 & 23 \\
\hline Plan de Formación & $\mathbf{3 0}$ & 54 \\
\hline
\end{tabular}

Fuente: Baro Duarte, J. y col.(2009)

Como se puede observar en la tabla, se muestra que existe una mayor dificultad en la implantación del Plan de trazabilidad y el Plan de formación, siendo mayor el número de establecimientos en los que no están implantados de los que sí. Esto se debe a que el plan de trazabilidad entraña mayor grado de complejidad, y las empresas en las que se pretende implantar constan de pocos recursos. En cuanto al 
Plan de formación, su falta de implantación radica en el hecho que los operarios entienden este plan con la mera obtención del Certificado de Formación en Higiene Alimentaria. El Plan de Control de Plagas también encuentra inconvenientes en su implementación, debido al concepto erróneo de vigilancia de plagas. Los restantes planes presentan un grado de implantación muy similar debido a su fácil ejecución y vigilancia (Baro Duarte, J. y col., 2009).

Un tercer estudio sobre la valoración de la implantación del APPCC en diferentes sectores alimentarios de Murcia, muestra los siguientes resultados: deficiencias detectadas en planes de trazabilidad de un $29 \%$ y documentación o registros no idóneos en un $44 \%$.

Los problemas de trazabilidad se atribuyen a la diversidad de la cadena alimentaria distribuidora, y la inadecuación del APPCC, es debida, entre otros, a la carencia de documentación idónea, revela dificultades para gestionar las auditorías externas (González Fernández, M. y col., 2007).

En otro de los estudios sobre la evolución de la implementación de los sistemas de autocontrol en 36 comedores colectivos, se puede observar que en el 2003 sólo 12 centros disponían de RPHT, y en 2007 la totalidad disponían de los requisitos previos, siendo el $28 \%$ de estos completo (APPCC y prerrequisitos) y el $72 \%$ sólo disponía de prerrequisitos. Dentro los requisitos previos, el Plan de Trazabilidad y el Plan de Mantenimiento de la Cadena de Frío no eran verificados. Por lo tanto, se concluye que en la actualidad los comedores colectivos disponen de Documentos de Autocontrol, no obstante, existe una gran variabilidad en el grado de implantación (Serrano Galán, E., 2009).

En general, tanto los resultados obtenidos a nivel de los planes generales de higiene y a nivel del APPCC fueron favorables. No obstante se observa que hay planes generales mejorables como: el plan de mantenimiento y la trazabilidad; y en el sistema APPCC: la gestión documental y la verificación (Villar Gaspar M.R., 2009).

Se ilustra un estudio en un matadero de ganado bovino (terneros) y porcino. En cualquier caso, son mayoritarios los fallos relacionados con la "Limpieza y desinfección" (de 14 fallos a 3) y "Mantenimiento de instalaciones y equipos" (de 13 a 2), refiriéndose en general al mal estado de conservación de las paredes interiores del matadero con defectos en el esmaltado, equipos con mantenimiento deficiente, con pérdida de galvanizado y óxido, superación de valores microbiológicos permitidos en tripería, esterilizador de cuchillos y paredes de la zona de sangrado así como en el mobiliario y en las canales (Suárez Iglesias, J.L. y col., 2012).

\section{Conclusión}

A modo de conclusión final, cabría resaltar que en el caso de los comercios minoristas son escasos aquellos que poseen sistemas de autocontrol, por lo que sería conveniente establecer criterios de flexibilidad en estos casos concretos. Por otra parte, en la mayoría de los casos estudiados (Baro Duarte, J. y col., 2009; González Fernández, M. y col., 2007; Serrano Galán, E., 2009; Villar Gaspar M.R., 2009), se evidencia que el plan de trazabilidad es el que presenta más complejidad a la hora de su desarrollo e implantación, debido a la limitación de recursos que presentan algunas empresas. En cuanto a la aplicación del propio sistema APPCC, es deficiente el punto de verificación y adopción de medidas correctoras ante desviaciones de PCC's. Por lo que sería recomendable un análisis profundo y el establecimiento de modelos que mejoren las garantías actuales. La aplicación de los modelos sistémicos podría ser una respuesta a las carencias detectadas. Los estudios emprendidos actualmente desde esta perspectiva deberán validar la propuesta sistémica y corroborar mediante resultados esta posibilidad.De forma global, los sistemas de autocontrol se encuentran, por tanto, dentro de los estándares de 
Evaluación y estudio comparativo mediante modelos sistémicos de la implantación del APPCC aplicado al sector agroalimentario.

conformidad necesarios para cubrir la exigencia legal impuesta por los Reglamentos Comunitarios en su aplicación.

Con todo esto, se pone en antecedentes la situación sobre la implantación de los sistemas de autocontrol en establecimientos agroalimentarios, y se procede a la recopilación de datos en algunos establecimientos que deberán mediante casos de estudio evaluar la implementación y resultados comparativos mediante el uso de modelos sistémicos.

\section{Referencias}

AESAN-MARM. Plan Nacional de Control Oficial de la Cadena Alimentaria 2010-2015.

ARJONA, C. y col. "Valoración de la implantación de planes generales de higiene (PGH) en minoristas del Aljarafe (Sevilla) Previa a la publicación de la guía de requisitos simplificados de higiene (RSH). Justificación de los RSH. López, A. (director) En: $3^{\circ}$ Congreso Internacional de Autocontrol y Seguridad Alimentaria (23-25 de abril 2008. Córdoba). Alimentaria Congresos año 2009 n² (pág. 6).

AVIÑÓ, JENARO y col.(2001).Plan de Seguridad Alimentaria de la Comunidad Valenciana. Ed. Generalitat Valenciana. Valencia

BARO DUARTE, J. y col. "Evaluación del grado de implantación de los sistemas de autocontrol en las industrias de harinas y derivados del área sanitaria norte de Córdoba". López, A. (director) En: $3^{\circ}$ Congreso Internacional de Autocontrol y Seguridad Alimentaria (23-25 de abril 2008. Córdoba). Alimentaria Congresos año 2009 n² (pág.22).

CARRAVILLA, S. y col. "Instrucción para la auditoría oficial de los establecimientos alimentarios en la comunidad de Madrid” López, A. (director) En: $3^{\circ}$ Congreso Internacional de Autocontrol y Seguridad Alimentaria (23-25 de abril 2008. Córdoba). Alimentaria Congresos año 2009 n² (pág.33).

CELAYA CARRILlo, C. (2004). Evaluación de la Implantación del Sistema de Análisis de Peligros y Puntos Críticos (APPCC) en pequeñas industrias alimentarias de la Comunidad de Madrid. Tesis Doctoral. Madrid. COMUNIDAD ECONOMICA EUROPEA.Reglamento (CE) n ${ }^{\circ}$ 178/2002 del Parlamento Europeo y del Consejo de 28 de enero de 2002 por el que se establecen los principios y los requisitos generales de la legislación alimentaria, se crea la Autoridad Europea de Seguridad Alimentaria y se fijan procedimientos relativos a la seguridad alimentaria.

COMUNIDAD EUROPEA.Reglamento (CE) no 852/2004 del Parlamento Europeo y del Consejo de 29 de abril de 2004 relativo a la higiene de los productos alimenticios.

COMUNIDAD EUROPEA.Reglamento (CE) no 853/2004 del Parlamento Europeo y del Consejo de 29 de abril de 2004 por el que se establecen normas específicas de higiene de los alimentos de origen animal.

COMUNIDAD EUROPEA.Reglamento (CE) no 854/2004 del Parlamento Europeo y del Consejo de 29 de abril de 2004 por el que se establecen normas específicas para la organización de controles oficiales de los productos de origen animal destinados al consumo humano.

COMUNIDAD EUROPEA. Reglamento (CE) no 882/2004 del Parlamento Europeo y del Consejo de 29 de abril de 2004 (controles oficiales y auditorías) sobre los controles oficiales efectuados para garantizar la verificación del cumplimiento de la legislación en materia de piensos y alimentos y la normativa sobre salud animal y bienestar de los animales.

COMUNIDAD EUROPEA.Reglamento (CE) no 2160/2003, de 17 de noviembre, sobre control de Salmonella y otros agentes zoonóticos transmitidos por los alimentos.

COMUNIDAD EUROPEA Reglamento (CE) no 1069/2009 del Parlamento Europeo y del Consejo, de 21 de octubre de 2009, por el que se establecen las normas sanitarias aplicables a los subproductos animales y los productos derivados no destinados al consumo humano y por el que se deroga el Reglamento (CE) n ${ }^{\circ}$ 1774/2002. 


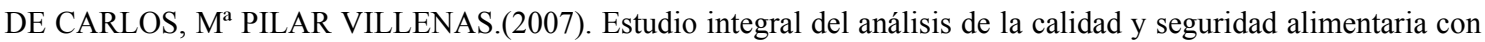
el fin de definir acciones estratégicas por parte de la industria agroalimentaria. Tesis Doctoral. Madrid

EUROPEAN COMISSION. (2011) Audit in order to evaluate the implementation of measures concerning officials controls on feed legislation. Final Report. Spain

ESPAÑA. Dirección General de Salud Pública. Consejería de Sanidad y Consumo de la Región de Murcia. (2010). Programa de Autocontrol Sanitario en Mataderos. Ed. Monografías sanitarias nº 27. Murcia

ESPAÑA.Real Decreto 1940/2004, de 27 de septiembre, sobre la vigilancia de las zoonosis y los agentes zoonóticos.

ESPAÑA. Ley 14/1986, de 25 de abril, General de Sanidad

ESPAÑA. Ley 8/2003, de 24 de abril, de Sanidad Animal

FRANCO PÉREZ, M.S. y col. "Autocontrol en la industria alimentaria: análisis de su situación en el departamento 14 de la Comunidad Valenciana”. López, A. (director) En: $3^{\circ}$ Congreso Internacional de Autocontrol y Seguridad Alimentaria (23-25 de abril 2008. Córdoba). Alimentaria Congresos año 2009 nº 2 (pág. 59).

GOMBAU, JUAN Y PALOMARES, SILVIA. (2011). Guías de Prácticas Correctas de Higiene del Sector de Mataderos y Ungulados Domésticos y Ratites. Ed. FEDACOVA. Valencia

GÓMEZ, E. "Programa piloto para la implantación de la guía APPCC en establecimientos de restauración BenidormCosta Blanca". López, A. (director) En: $3^{\circ}$ Congreso Internacional de Autocontrol y Seguridad Alimentaria (23-25 de abril 2008. Córdoba). Alimentaria Congresos año 2009 n² (pág.31).

GONZÁLEZ FERNÁNDEZ, M. y col. (2007) "Valoración de la implantación del APPCC en sectores alimentarios en CCAA de Murcia”. López, A. (director) En: $3^{\circ}$ Congreso Internacional de Autocontrol y Seguridad Alimentaria (23-25 de abril 2008. Córdoba). Alimentaria Congresos año 2009 nº2 (pág. 27).

GUTIÉRREZ, N. y col (2011) Evaluación de prerrequisitos en el sistema HACCP en empresas del sector agroalimentario. [Consulta 10/07/2016]

HERNANDIS, B. (2003). Tesis Doctoral: Desarrollo de una metodología sistémica para el diseño de productos industriales. . Valencia, España: Universidad Politécnica de Valencia.

MARTÍ, L.E. y col. (2012) "Food Safety. La seguridad alimentaria como política pública" Organización Panamericana de Salud/ Organización Mundial de la Salud (OPS/OMS).

MEJÍAS CARPENA, C. y col. "Implantación del sistema de autocontrol en la pequeña industria ubicada en el ámbito rural" López, A. (director) En: $3^{\circ}$ Congreso Internacional de Autocontrol y Seguridad Alimentaria (23-25 de abril 2008. Córdoba). Alimentaria Congresos año 2009 n² (pág.33).

SERRANO GALÁN, E. "Evolución de la implementación de los sistemas de autocontrol en comedores colectivos". López, A. (director) En: $3^{\circ}$ Congreso Internacional de Autocontrol y Seguridad Alimentaria (23-25 de abril 2008. Córdoba). Alimentaria Congresos año 2009 n² (pág. 36).

SUÁREZ IGLESIAS, J.L. y col. (2012) "Estudio de la eficacia de los sistemas APPCC para detectar deficiencias: caso de un matadero de bovino y porcino". Eurocarne $\mathrm{N}^{\circ}$ 203. Enero-Febrero 2012. Laboratorio de Higiene, Inspección y Control de Alimentos. Facultad de Veterinaria, USC. 27002, Lugo.

SUASNAVAS, N. y col. (2007) Requerimientos técnicos para la implementación del Sistema de Análisis de Peligros y Puntos Críticos de Control (HACCP) en un matadero porcino. $<$ http://www.veterinaria.org/revistas/redvet/n080807/080706.pdf $>$ [Consulta 08/07/2016]

STUART A. SLORACH. (2002). Enfoques integrados para la gestión de la inocuidad de los alimentos a lo largo de toda la cadena alimentaria. Foro Mundial FAO/OMS de las Autoridades de Reglamentación sobre Inocuidad de los Alimentos. Suecia. 
Evaluación y estudio comparativo mediante modelos sistémicos de la implantación del APPCC aplicado al sector agroalimentario.

VILLAR GASPAR M.R. "Auditorías en actividades de restauración sin registro sanitario de alimentos en un distrito de Madrid”. López, A. (director) En: $3^{\circ}$ Congreso Internacional de Autocontrol y Seguridad Alimentaria (23-25 de abril 2008. Córdoba). Alimentaria Congresos año 2009 n²2 (pág. 79).

CELAYA CARRILlO, C. (2004). Evaluación de la Implantación del Sistema de Análisis de Peligros y Puntos Críticos (APPCC) en pequeñas industrias alimentarias de la Comunidad de Madrid. Tesis Doctoral. Madrid.

MARTÍ, L.E. y col. (2012) "Food Safety. La seguridad alimentaria como política pública" Organización Panamericana de Salud/ Organización Mundial de la Salud (OPS/OMS).

SUÁREZ IGLESIAS, J.L. y col. (2012) "Estudio de la eficacia de los sistemas APPCC para detectar deficiencias: caso de un matadero de bovino y porcino". Eurocarne N ${ }^{\circ}$ 203. Enero-Febrero 2012. Laboratorio de Higiene, Inspección y Control de Alimentos. Facultad de Veterinaria, USC. 27002, Lugo. 